Sonderdruck aus

Forstwissenschaftliches Centralblatt

92. Jahrgang (1973), H. 3, S. 105-111

Alle Rechte, auch die der Ubersetzung, des Nachdrucks, der photomechanischen Wiedergabe und Speicherung in Datenverarbeitungsanlagen, vorbehalten.

(c) 1973 Verlag Paul Parey, Hamburg und Berlin

\title{
Nr. 106, 1973

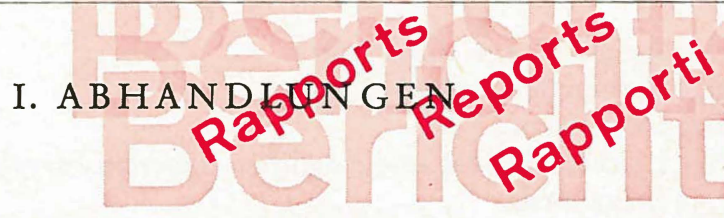 Über den Einfluß von Industrieklärschlamm auf die
Nettoassimilation junger Forstpflanzen
}

Von TH. KeLLER

\section{Einleitung und Fragestellung}

Die Reinhaltung öffentlicher Gewässer ist ein dringendes Gebot der Stunde. In der Folge sind zahlreiche Kläranlagen entstanden oder im Bau begriffen. Aber nicht nur die häuslichen Abwässer bedürfen der Reinigung, sondern auch die mannigfaltigen industriellen Abwässer verschiedenster chemischer Zusammensetzung. Der in den Klärbecken anfallende Schlamm wird im Faulturm in der Regel anaerob ausgefault, wobei die Abwasserfachleute diesem Fäuleprozeß vollste Beachtung schenken, denn jede bedeutende, für die Bakterien giftige Beimengung im Schlamm vermag diesen Prozeß zu beeinträchtigen oder gar lahmzulegen. Aus diesem Grunde wird auch behauptet, ausgefaulter Klärschlamm sei für Pflanzen völlig unschädlich.

Mit der steigenden Zahl von Kläranlagen bei stetig abnehmender Bodenfläche der Urproduktion beginnt der Absatz für den Klärschlamm da und dort zu stocken und vor allem dem Industrieklärschlamm begegnet man weitherum mit Skepsis. Die Entwässerung und Verbrennung der gewaltigen anfallenden Schlammengen ist jedoch kostspielig und kann mit lästigen oder gar schädlichen Immissionen verbunden sein. Deshalb versucht man, möglichst allen Klärschlamm auf Grünflächen auszubringen.

Die meisten Forstleute schrecken vor einer Ausbringung von Klärschlamm im Wald zurück, denn gerade die stadt- und industrienahen Wälder üben die Funktion von Naherholungsgebieten aus. Diese Funktion würde durch die evtl. mit Geruchsbelästigungen verbundene und nicht ästhetische Beschickung mit Klärschlamm zeitweilig stark beeinträchtigt. Zudem ist völlig unbekannt, wie eine so komplexe Biocoenose wie ein Waldbestand auf einen derartigen Eingriff reagiert. Ist eine Klärschlammgabe mit einer Düngung vergleichbar oder sind auch toxische Komponenten mit im Spiele, welche z. B. die Mykorrhiza oder physiologische Funktionen negativ beeinflussen?

$\mathrm{Da}$ Versuche in den letzten Jahren gezeigt hatten, daß insbesondere die Photosynthese (Nettoassimilation) auf Veränderungen der Umwelt, wie Düngung, Biozideinsatz etc. (vgl. z. B. Keller und КосH, 1962; Keller, 1964, 1969; KozlowsKI und KeLLER, 1966), empfindlich reagiert, wurden im Sommer 1969 zwei Versuche mit Topfpflanzen durchgeführt, welche den Einfluß einer Klärschlammbehandlung auf die Nettoassimilationsleitung einiger Baumarten zeigen sollten. 


\section{Material und Methodik}

\subsection{Material}

Zur Verfügung standen je 10 vertopfte

4jährige Fichten und Buchen (2/2),

3jährige Douglasien (2/1),

2jährige Lärchen, Kiefern und Roteichen (Quercus borealis maxima) (1/1),

1jährige Bergahorne.

Von der Eidg. Anstalt für Wasserversorgung, Abwasserreinigung und Gewässerschutz wurde Industrie-Klärschlamm zur Verfügung gestellt ${ }^{1}$. Dessen chemische Analyse ergab:

\begin{tabular}{|c|c|c|c|}
\hline $\begin{array}{l}\text { Trockensubstanz (TS) } \ldots \\
\text { pH-Wert } \ldots \ldots \ldots \ldots \ldots \\
\text { organ. C } \ldots \ldots \ldots \ldots \ldots \\
\mathrm{N} \text { (total) } \ldots \ldots \ldots \ldots \ldots\end{array}$ & $\begin{array}{l}2,3 \% \\
6,4 \\
38,3 \% \text { der TS } \\
9,1 \% \text { der TS }\end{array}$ & 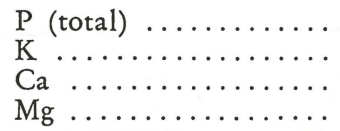 & $\begin{array}{l}0,4 \% \text { der TS } \\
0,2 \% \text { der TS } \\
1,2 \% \text { der TS } \\
0,3 \% \text { der TS }\end{array}$ \\
\hline
\end{tabular}

\subsection{Methodik}

Von jeder Art wurde die Hälfte der Pflanzen zur Behandlung ausgelost. Jeder Topf erhielt $2 \times 2,2 \mathrm{dl}$ Klärschlamm und dazwischen $2 \mathrm{dl}$ entsalztes Wasser. Dies entsprach einer Klärschlammgabe von $220 \mathrm{~m}^{3} / \mathrm{ha}(22 \mathrm{~mm})$. Der Klärschlamm war sehr ölig, versickerte schlecht und bildete eine schwarze, gummiartige Kruste an der Bodenoberfläche, die nach wenigen Tagen aufriß.

Die unbehandelten Nullproben erhielten $220 \mathrm{~m}^{3} / \mathrm{ha}$ entsalztes Wasser $(4,4 \mathrm{dl} / \mathrm{Topf})$.

Beim 2. Versuch, diesmal mit Kiefer und Bergahorn durchgeführt, wurde der Klärschlamm nicht oberflächlich appliziert. Statt dessen wurden die Pflanzen mit dem gesamten Wurzelballen aus dem Topf gehoben, der ganze Ballen 5 Minuten in den Klärschlamm getaucht und nachher in einen größeren Topf neu gesetzt. Die Nullproben wurden gleichlang in entsalztes Wasser getaucht, um den Wurzelballen zu sättigen. Beim 2. Versuch gelangte ein Teil des Wurzelwerkes (Topfballenoberfläche) in direkten Kontakt mit dem unverdünnten Klärschlamm.

Die Messung der Nettoassimilation erfolgte im Labor gemäß bereits beschriebener Methodik (vgl. Keller, 1966) bei $30 \mathrm{kLux}$. Als Lichtquelle diente eine Osram-XenonHochdrucklampe (6000 Watt). Der Luftdurchsatz durch jede Küvette betrug am Versuchsanfang 10-20 1/min, später 15-30 1/min und richtete sich nach Größe und Aktivität der Pflanzen.

Die Assimilationsleistung unmittelbar vor der Behandlung wurde als Vergleichsbasis benutzt und für jede Pflanze gleich 100\% gesetzt. Die Assimilationsleistung bleibt ja während der Vegetationsperiode nicht konstant, einmal wegen der Reifung und Alterung der Assimilationsorgane, aber auch wegen der ständigen Neubildung von Assimilationsorganen bei Laubbaumarten (vgl. Abb. 1). Um nun zur Charakterisierung der Reaktion der Assimilation auf die Behandlung nicht für jede Baumart zwei Kurven aufzeichnen zu müssen, wurde das Verhältnis relative Nettoassimilation der behandelten Pflanzen : relative Nettoassimilation der unbehandelten Pflanzen für jeden Meßtag berechnet. Ein Quotient $>1$ bedeutet eine Assimilationszunahme gegen-

1 Ich danke Herrn Dipl.-Ing. agr. A. von HirschHeyd für die Bereitstellung von Klärschlamm und Analysendaten. 
über der Nullprobe, ein Quotient $=1$ bedeutet keine Reaktion und ein Quotient $<1$ bedeutet eine negative Reaktion, nämlich eine Einbuße der Assimilationsleistung (vgl. Abb. 2-4).

Die Chlorophyllgehaltsbestimmung erfolgte in $80 \%$ Azeton nach ZIEGLER und EGLE (1965).

\section{Resultate}

Abb. 1 zeigt den zeitlichen Verlauf der relativen Nettoassimilation von Fichte und Kiefer. Mit fortschreitender Nadelentwicklung steigt die Assimilation der Nullproben an und erreicht bei der Fichte nach $21 / 2$ Monaten rund das 31/2fache der anfänglichen Leistung. Bei der Kiefer ist die Zunahme wesentlich bescheidener. Der markanteste Unterschied zwischen den beiden Arten ist jedoch die Tatsache, daß die Assimilation der Fichte durch die Klärschlammbehandlung gesteigert wurde (Kurve der behandelten Pflanzen liegt über der Kurve der Nullproben), wogegen die Verhältnisse bei der Kiefer gerade umgekehrt liegen. Die Entwicklung der Kiefer wurde durch den Klärschlamm derart gehemmt, daß auch 3 Wochen nach der Behandlung noch keine wesentliche Assimilationszunahme festzustellen war. Die Nullproben dagegen wiesen eine rund 50prozentige Steigerung auf.

In Abb. 2 ist der Verlauf des Quotienten für Kiefer und Douglasie dargestellt. Bei der Kurve für Kiefer kommt die oben beschriebene Hemmung der Assimilation deutlich zum Ausdruck, bleibt der Quotient doch für die ganze untersuchte Zeitspanne deutlich $<1$. Der Kurvenverlauf deutet an, daß sich die Verhältnisse noch für längere Zeit kaum ändern werden. Bei Douglasie ist anfänglich eine vorübergehende Stimulierung festzustellen, die jedoch sehr rasch abklingt. Nach 2-3 Wochen zeigt sich die Tendenz einer neuerlichen Stimulierung.

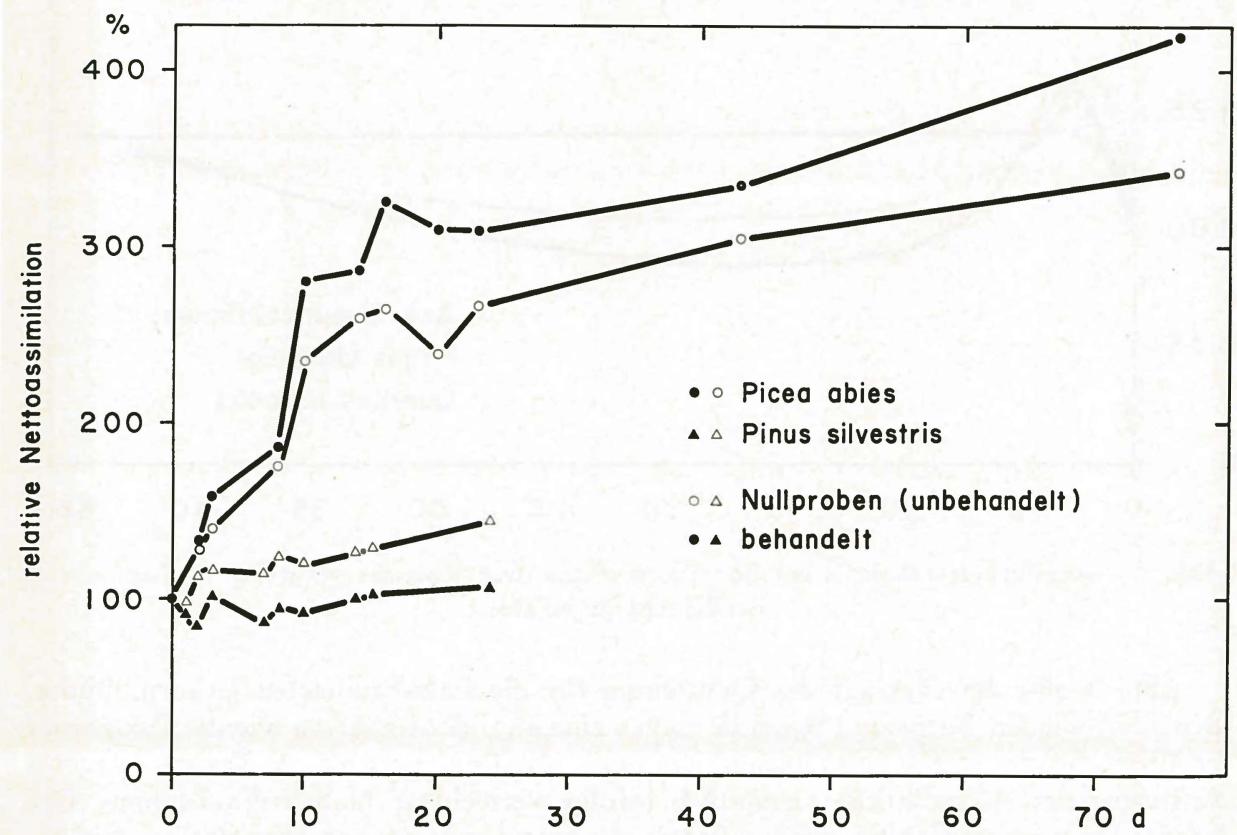

Abb. 1. Relative Nettoassimilation von Fichte und Kiefer, mit und ohne Klärschlammbehandlung 
Verhältnis behandelt / unbehandelt

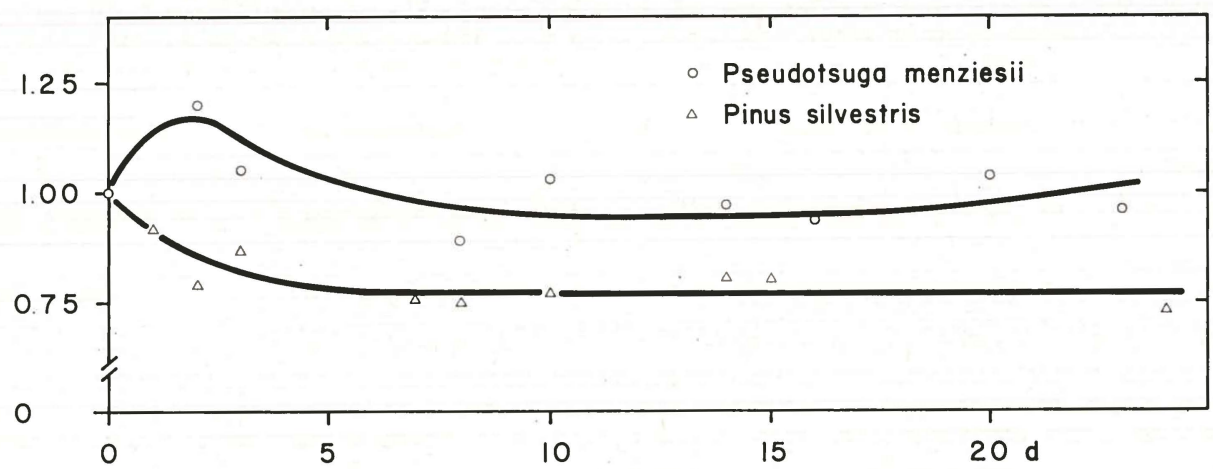

Abb. 2. Verhältnis der Assimilation behandelter zu derjenigen unbehandelter Kiefern und Douglasien während 24 Tagen nach der Klärschlammbehandlung

Die anfängliche Stimulierung, die übrigens auch bei Buche und Roteiche sehr ausgeprägt auftrat (Abb. 3), können wir nicht erklären. Die spätere Tendenz einer neuerlichen Stimulation dürfte durch eine vermehrte Nährstoffaufnahme (Düngewirkung) bedingt sein, denn die nach Abschluß der Versuche (3 Monate nach Behandlung) durchgeführte Chlorophyllgehaltsbestimmung zeigte bei sämtlichen Arten eine Chlorophyllzunahme durch die Behandlung.

\section{Verhältnis behandelt / unbehandelt}

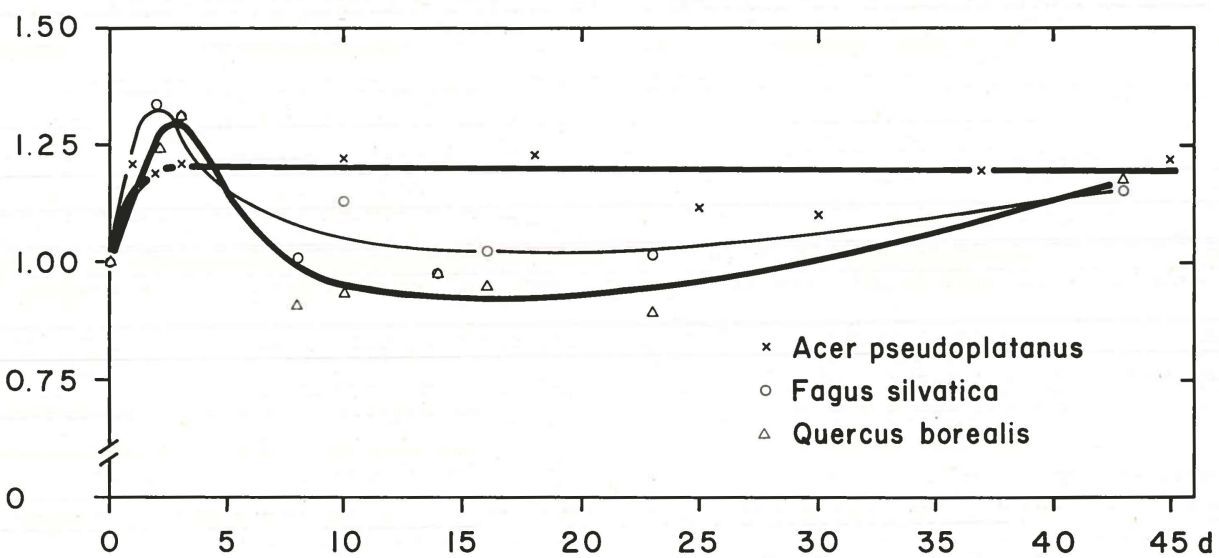

Abb. 3. Assimilationsverhältnis bei Bergahorn, Buche und Roteiche während 45 Tagen nach der Klärschlammgabe

Abb. 3 gibt den Verlauf des Quotienten für die Laubbaumarten (Ahorn, Buche, Roteiche) wieder. Buche und Roteiche zeigen eine ähnliche Reaktion wie die Douglasie: vorübergehende, starke Stimulation, die rasch abklingt, nach ca. 3 Wochen erneutes Ansteigen der Assimilation, vermutlich infolge vermehrter Nährstoffaufnahme. Der Bergahorn reagierte völlig anders, indem die sofort einsetzende Stimulation den behandelten Pflanzen einen Assimilationsvorsprung sicherte, der während Wochen konstant blieb. Die Beobachtung, daß die Ahornblätter sehr rasch dunkelgrün wurden, 
Verhältnis behandelt / unbehandelt

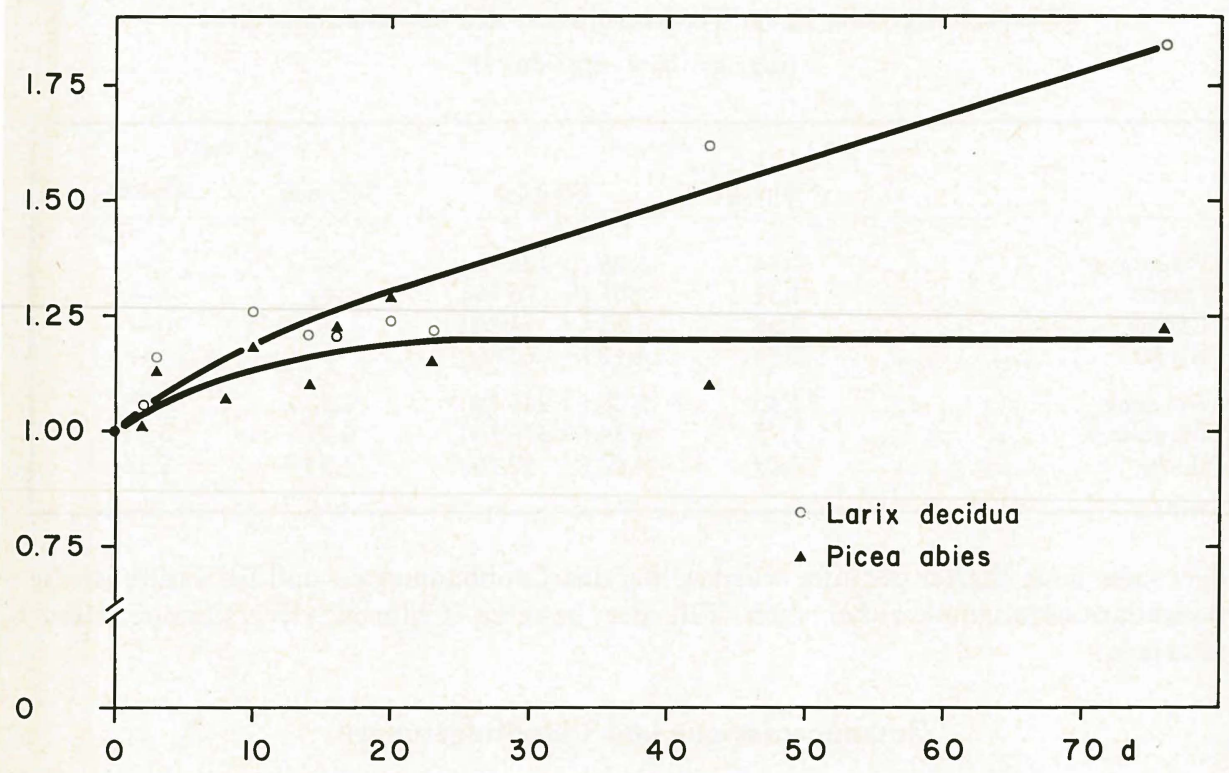

Abb. 4. Assimilationsverhältnis bei Lärche und Fichte während 73 Tagen nach der Klärschlammbehandlung

aber klein blieben, deutet darauf hin, daß die Stimulierung der Chlorophyllbildung eher einem Spurenelement als dem Stickstoff des Klärschlamms zuzuschreiben ist.

Abb. 4 endlich zeigt die Verhältnisse bei Lärche und Fichte. Beide Arten reagierten positiv auf den Klärschlamm. Die Fichtenkurve ähnelt der Kurve für Bergahorn (Abb. 3), nur daß die Stimulation der Assimilation wesentlich langsamer erfolgte. Nach ca. 4 Wochen blieb der Quotient konstant, d.h. die Assimilationssteigerung blieb erhalten (vermutlich dank erhöhtem Chlorophyllgehalt der Nadeln). Bei der Lärche dagegen hielt die Assimilationssteigerung über Wochen hinweg an. Bei dieser Art wirkte sich die Klärschlammgabe offensichtlich düngend aus. Der Stoffgewinn wurde laufend in neue Assimilationsorgane investiert, was die nachhaltige Steigerung ermöglichte. Am Ende der Vegetationsperiode war der Längstrieb der behandelten Lärchen doppelt so lang wie jener der Nullproben. Die übrigen Arten wiesen keine statistisch gesicherten Unterschiede im Längenwachstum auf.

In Tabelle 1 sind die Ergebnisse der Chlorophyllgehaltsbestimmungen, die Mitte August vorgenommen wurden, zusammengestellt.

Aus Tabelle 1 geht hervor, daß gerade die Nadelbaumart mit dem stärksten Chlorophyllzuwachs, die Douglasie, assimilatorisch kaum reagierte (es wurde offenbar ein Pigmentüberschuß geschaffen, der gar nicht zur Wirkung kam, weil vermutlich ein anderer Faktor limitierend wirkte); ähnlich liegt der Fall bei der Kiefer (vgl. Abb. 2). Möglicherweise läßt sich die Diskrepanz zwischen den Ergebnissen der Assimilationsmessung und der Chlorophyllbestimmung aber auch damit erklären, daß die Assimilationsmessung bereits nach wenigen Wochen abgebrochen wurde. Bei der Lärche trat keine wesentliche Erhöhung des Chlorophyllgehaltes der Nadeln ein, weil der Zuschuß ständig für die neugebildeten Nadeln verbraucht wurde. Beim Bergahorn dagegen zeigt sich ein wesentlich größerer Chlorophyllgewinn pro Flächeneinheit als pro Gewichtseinheit, da, wie schon erwähnt, die Blätter klein blieben und auch nicht beson- 


\section{Tabelle 1}

Chlorophyllgehalte in der Trockensubstanz der Assimilationsorgane

$\left(\mathrm{mg} \cdot \mathrm{g}^{-1}\right.$ bzw. $\left.\mathrm{mg} \cdot \mathrm{dm}^{-2}\right)$

\begin{tabular}{|c|c|c|c|c|}
\hline \multirow{2}{*}{ Art } & \multicolumn{2}{|c|}{$\mathrm{mg} \cdot \mathrm{g}-1$} & \multicolumn{2}{|c|}{$\mathrm{mg} \cdot \mathrm{dm}-2$} \\
\hline & Nullprobe & behandelt & Nullprobe & behandelt \\
\hline Douglasie ... & 1,44 & $3,25(+126 \%)$ & - & - \\
\hline Fichte ... & 1,51 & $2,65(+75 \%)$ & - & - \\
\hline Lärche ... & 2,66 & $3,03(+14 \%)$ & - & - \\
\hline Kiefer & 2,75 & $4,46(+62 \%)$ & - & - \\
\hline . $\ldots \ldots \ldots \ldots \ldots$ & 1,45 & $5,00(+245 \%)$ & 0,80 & 2,75 \\
\hline Bergahorn & 1,75 & $8,34(+376 \%)$ & 0,94 & 5,31 \\
\hline Buche .. & 2,09 & $4,00(+92 \%)$ & 1,13 & 2,16 \\
\hline
\end{tabular}

ders viele neue Blätter gebildet wurden. Bei den Laubbaumarten und bei Fichte ist die Assimilationszunahme sicher zum Teil der besseren Chlorophyllversorgung zuzuschreiben.

\section{Zusammenfassung und Schlußfolgerungen}

Der Einfluß einer Klärschlammgabe im Ausmaß von $220 \mathrm{~m}^{3} / \mathrm{ha}$ auf die Nettoassimilation (Infrarot-Gasanalyse) und den Chlorophyllgehalt von vertopften Jungpflanzen der Arten Fichte, Kiefer, Lärche, Douglasie, Buche, Roteiche und Bergahorn wurde im Labor untersucht.

Die Arten reagierten unterschiedlich: eine starke Stimulierung der Assimilation (die sich auch in vermehrtem Höhenzuwachs äußerte) wurde bei der Lärche festgestellt. Auch bei Fichte und Bergahorn war sofort eine anhaltende Steigerung der Assimilation erfaßbar. Bei Buche, Roteiche und Douglasie zeichnete sich erst nach einigen Wochen eine Stimulation der Assimilation ab, die mit erhöhtem Chlorophyllgehalt zusammenfiel. Die Kiefer reagierte während der Versuchsperiode mit einer deutlichen Assimilationsdepression.

Bei einer Klärschlammausbringung im Walde ist daher mit einer von Art zu Art unterschiedlichen Reaktion (auch negativer Art) zu rechnen.

Abschließend danke ich den Herren S. Marjanovic und H. Schwager für gewissenhafte Mithilfe und Betreuung des Versuches.

\section{Summary}

The effect of an application of industrial sewage sludge $\left(220 \mathrm{~m}^{3} / \mathrm{ha}\right)$ on net photosynthesis (infrared gas analysis) and chlorophyll content of potted young spruces, pines, larches, Douglas firs, beeches, Northern red oaks and sycamores was investigated in the laboratory.

The species investigated differed in reaction: larch showed a strong stimulation of net photosynthesis (and of shoot length). Also in spruce and sycamore an immediate and sustained increase of $\mathrm{CO}_{2}$-uptake was detected. Beech, red oak and Douglas fir reacted quickly (but for a few days only) with a stimulation of photosynthesis. Several weeks later photosynthesis tended to increase again, probably due to increased 
chlorophyll content. Pine reacted with a distinct depression of net $\mathrm{CO}_{2}$-uptake over several weeks.

The sewage application in a forest thus will differ in effect with species and may even be negative.

\section{Résumé}

L'influence d'un épandage de boue d'épuration, à raison de $220 \mathrm{~m}^{3}$ à l'ha, sur l'assimilation nette (infrarouge-analyse des gas) et la teneur en chlorophylle de jeunes épicéas, pins, mélèzes, douglas, hêtres, chênes rouges et érables de montagne en pots a été observée en laboratoire.

Les espèces réagirent différemment: une forte stimulation de l'assimilation (qui se manifesta aussi dans une augmentation de l'accroissement en hauteur) fut constatée chez le mélèze. Chez l'épicéa et l'érable de montagne, une hausse continue de l'assimilation fut également enregistrée.

Pour l'hêtre, le chêne rouge et le douglas, une stimulation de l'assimilation ne se dessina qu'après quelques semaines et coïncida avec une teneur en chlorophylle accrue. Les pins montrèrent une dépression d'assimilation évidente au cours de la période d'essai.

Lors d'un épandage en forêt, une réaction différente d'espèce à espèce (aussi d'effet négatif) est donc à prendre en considération.

Trad.: S. KOBERT

\section{Literatur}

Keller, TH., 1964: Beeinflussen Insektizide die Photosynthese und Transpiration von Pappelblättern? Anz. Schädlingskunde 37, 87-89. - KelleR, TH., 1966: Über den Einfluß von transpirationshemmenden Chemikalien (Antitranspirantien) auf Transpiration, $\mathrm{CO}_{2}$-Aufnahme und Wurzelwachstum von Jungfichten. Forstw. Cbl. 85, 65-79. - Keller, TH., 1969: The influence of fertilization on gaseous exchange of forest tree species. In: Forest Fertilization Walddüngung. Internat. Kali-Inst. Bern. Jyväskylä p. 65-79. - Keller, TH.; Koch, W., 1962: Der Einfluß der Mineralstoffernährung auf $\mathrm{CO}_{2}$-Gaswechsel und Pigmentgehalt der Pappel. Mitt. Schweiz. Anst. forstl. Vers.wesen 38, 253-318. - KozlowsKI, T. T.; Keller, TH., 1966: Food relations of woody plants. Bot. Rev. 32, 293-382. - ZIEGLER, R.; EgLE, K., 1965: Zur quantitativen Analyse der Chloroplastenpigmente I. Beiträge Biol. Pflz. 41, 11-37.

Anschrift des Verfassers: Dr. TH. Keller, Eidgenössische Anstalt für das Forstliche Versuchswesen, $\mathrm{CH}-8903$ Birmensdorf 